\title{
INFLUENCE OF MATERNAL FACTORS ON GROWTH PARAMETERS IN LOW-BIRTH-WEIGHT BABIES WITH KANGAROO MOTHER CARE
}

\author{
Lumbanraja SN \\ Department of Obstetrics and Gynecology, University of Sumatera Utara, Medan, Indonesia
}

\begin{abstract}
Background: Kangaroo mother care (KMC) in low birth weight newborns has been found to be beneficial, but studies have shown that maternal factors might be of concern in the successful application of KMC.
\end{abstract}

Aim: To study the influence of maternal factors on growth parameters in low-birth-weight babies with KMC.

Methods: This is a prospective cohort study of 40 low birth weight newborns in our institutions. We randomly assigned the newborns to the group which received KMC and to the group which received conventional care. Maternal factors were recorded. We measured weight, length, and head circumferences of newborns daily for thirty days. Data was processed by SPSS x22.0.

\begin{abstract}
Results: A total of 40 newborns were recruited into the study. Weight parameters were significantly higher in the KMC group than in the conventional group except for the $Z$ scores. Regarding maternal characteristics, only gestational age was found to influence the initial and the last head circumference $(p=0.035)$. There were no differences in maternal age, parity, maternal education, mode of delivery, fetal sex, and initial Apgar score with any of the growth parameters.
\end{abstract}

Conclusion: There were no maternal and fetal differences in the growth parameters of the groups, except in the delayed growth of head circumferences in preterm infants.

Keywords: Growth parameters, KMC method, low birth weight

\section{Introduction}

Low birth weights (LBW) in newborns is one of the leading causes of neonatal morbidity and mortality. As many as $16 \%$ of newborns worldwide have low birth weights with an annual incidence of 18 million. In developing countries, the incidence of LBW was two times higher than in developed countries (1). LBW is associated with many complications such as growth and cognitive disorders, and the development of chronic diseases of the elderly later in life (2).

Maternal factors such as age, multigravidity, narrow pregnancy interval, socio-economic factors, a low level of education, and weight gain during pregnancy increased the incidence of LBW (3-5). Other studies showed a 1.2 times increased risk of low birth weight in women with low socioeconomic status, and 1.7 times increased risk in women with low levels of education (6). LBW is also closely linked with maternal height (7) Mumbare et al. (8) by logistic regression showed that maternal weight $<55 \mathrm{~kg}$ (OR 4.81, 95\% Cl 2.53-9.15), maternal height $<145 \mathrm{~cm}$ (OR $4.13,95 \% \mathrm{Cl}$ 2.04-8.37), and inadequate antenatal care (OR $4.98,95 \% \mathrm{Cl}$ 2.64-9.39), were the most significant factors associated with LBW. Taha et al. (9) by logistic regression showed that only maternal age and parity had significant effects on birth weight ( $p=0.014$ and $p=0.008$ ). However, Negi et al. (10) on the other hand showed that only the primigravida had a higher risk of having an LBW baby (OR $3.21 ; p<0.01$ ) and found no relationship between maternal age, maternal height and weight with LBW. In Indonesia, Trihardani et al. (11) showed that the risk factors associated with LBW were maternal body mass index (RP 5.4; $95 \mathrm{Cl}$ 1.07-27.29), weight gain during pregnancy (RP 6.6; $95 \mathrm{Cl}$ 1.30-33.01), and parity ( $R P=5.30 ; 1.24-22.56)$ while there was no significance between in maternal age, maternal height, frequency of antenatal care, and pregnancy interval, with LBW.

Pregnancy in women who were under 20 years of age or who were over 35 years old had an unmet need of adequate nutrition for fetal growth (12). A too narrow pregnancy interval also indicated a shared nutritional condition at risk; and an inadequate time for the recovery of the reproductive system so that the following fetal birth weight in utero would be affected (13).

LBW infants should be kept warm because of their vulnerability to hypothermia which could lead to lifethreatening infection, apnea or massive bleeding (14). An incubator is a way to provide warmth for the LBW babies but it may prevent early maternal contact and 
breastfeeding (15). Invasive procedures in the incubator also induced stress in babies as proven by some studies that showed increasing heart rates and breaths in infants treated in an incubator (16). Inspired by how marsupials keep their newborns warm, KMC was developed by Martinez and Rey in Bogota (1978)(17).

KMC is effective in the treatment of low birth weight newborns. It is also easy to apply and it is inexpensive. It is very important in many remote areas in Indonesia due to the lack of modern health technology for resuscitation facilities (18). The majority of studies showed better results in infants treated with KMC than with the conventional method, although some of these results are still controversial (19-21). Pratiwi et al. (22) showed that the $B B L \geq 1500 \mathrm{~g}$ (RR 0.4; 95\%Cl 0.23-0.73; $p=0.001)$ and a neonatal age at the commencement of KMC > 10 days (RR 2.69; 95\% Cl 1:14 to $6: 32 ; \mathrm{P}=0.003$ ) were related to the successful use of KMC. Gestational age $\geq 34$ weeks (RR 0.94; 95\% Cl 0.46-1.89; $p=1.00$ ), KMC duration $\geq 65$ minutes $(p=0.215)$, and a high maternal education level $(p=0.408)$ did not influence the incidence of LBW. There is no study that specifically assessed the maternal factors that contribute to the success of KMC in LBW. Neonatalogists should be able predict the benefits at the beginning of a therapy because the neonatal period is the most vulnerable period in life and clinicians should apply a therapy that is of benefit.

The aim of the study was to determine the influence of maternal factors on growth parameters in low-birth-weight babies on KMC.

\section{Methods}

\section{Study design}

This was an analytical prospective cohort study that was conducted at the Adam Malik General Hospital and the Pirngadi Hospital, in Medan, Indonesia, from June to November 2015. Written informed consents were obtained from all parents. This study was approved by the Ethical Committee of University of Sumatera Utara.

\section{Study population}

In order to have a representative sample, we calculated the minimal sample size, and 20 babies were required in each study group. Forty consecutive low birth weight newborns, who were delivered in the two institutions, were enrolled into the study. A low birthweight is defined as a weight below 2500 g.The newborns were randomly assigned to the two groups. Randomization was done by computer generated-random numbers. In the first group, the newborn was given $\mathrm{KMC}$ and in the second group, they were cared in an incubator as the conventional method. This study only included babies with birth weight of 1000-2500 g, who were hemodynamically stable, who did not require oxygen therapy for most of the day, who had no need of continuous intravenous fluids, and with a mother who was healthy and willing to practice
KMC. This study excluded mothers who were exclusively breastfeeding because, from prior studies, breastfeeding could significantly affect KMC outcome. Exclusion criteria were patients who withdrew from the study or who were lost to follow-up, babies with congenital anomalies, severe perinatal complications that required NICU care, malignancies, metabolic and cardiovascular disorders and death.

\section{Study protocols}

The baseline information of maternal and fetal demographics was recorded. Maternal factors included maternal age, gestational age, the number of parity, maternal education, and mode of delivery. This study also included fetal demographics such as fetal sex and the initial Apgar Score.

In the conventional group, the babies were placed in an incubator, according to the -standard guidelines of the hospitals. In the KMC group, KMC therapy was initiated as soon as the babies were stable. At enrolment, the mothers were taught how to practice KMC. Mothers were seated in a comfortable chair close to the babies' cradle. They were shown how to hold their babies vertically, strapped to the middle of their (mothers') chests, with skin to skin contact so that the babies' skins were touching their mothers' skin. At other times, when not on KMC, the babies were placed in the cradle with their bodies covered. KMC was applied for 4-6 hours each day. Babies in both the groups were provided with vitamin and mineral supplementation as per the protocol.

The subjects were followed up for 30 days, with daily anthropometric measurements. Babies were weighed naked on an electronic weighing scale (GEA). The weighing machine was calibrated daily. The lengths were measured with an infantometer (GEA) at birth, day 10, day 20, and day 30 . The head circumferences were measured with a standard tape (Butterfly) at birth, day 10, day 20, and day 30. All measurements were carried out by two blinded investigators. The mean of both measurements was taken. During the follow-up, if there were sucking disorders, breathing disorders, or loss of consciousness, the babies were treated appropriately and were excluded from the study.

The babies' weights were plotted using Fenton's growth charts. We calculated the accurate weight gain velocity with the following formula GV $=[1000 \times(\mathrm{Wn}-\mathrm{W} 1)]$ : [(DnD1) $\times(W n+W 1) / 2$ ] and the estimated weight gain velocity with the formula GV [1000 x In (Wn/W1)] : (Dn-D1). The magnitude of errors was reflected in the percentage of absolute difference with the formula $100 \times$ (Estimated GV - Accurate GV) : Accurate GV. We also calculated a z-score for weight in www.peditools.org/fenton/2013 at birth and day 30 .

\section{Data analysis}

Data were analyzed by SPSS (Statistical Product and Service Solutions, Chicago, IL, USA) 22.0 for Windows. Categorical data were expressed as number and continuous data as 
mean \pm SD. Chi-square test (Fisher's exact test) was used to examine the relation between qualitative variables. T-independent, T-dependent, Pearson correlation were used to evaluate quantitative variables. The significance was taken at $95 \%$ with a $p$-value $<0.05$.

\section{Results}

During the study period, from June to October 2015, there were 90 babies who were born with a low birth weight.
Two mothers were not willing to participate in KMC. Some newborns were excluded from the study. There were 45 newborns who needed NICU care; of these, 1 had a congenital anomaly, 1 with a cardiovascular disorder, and 1 was a newborn who was dead. 40 newborns were eligible for the study and were successfully enrolled. There was no loss to follow up or from withdrawal from the study.

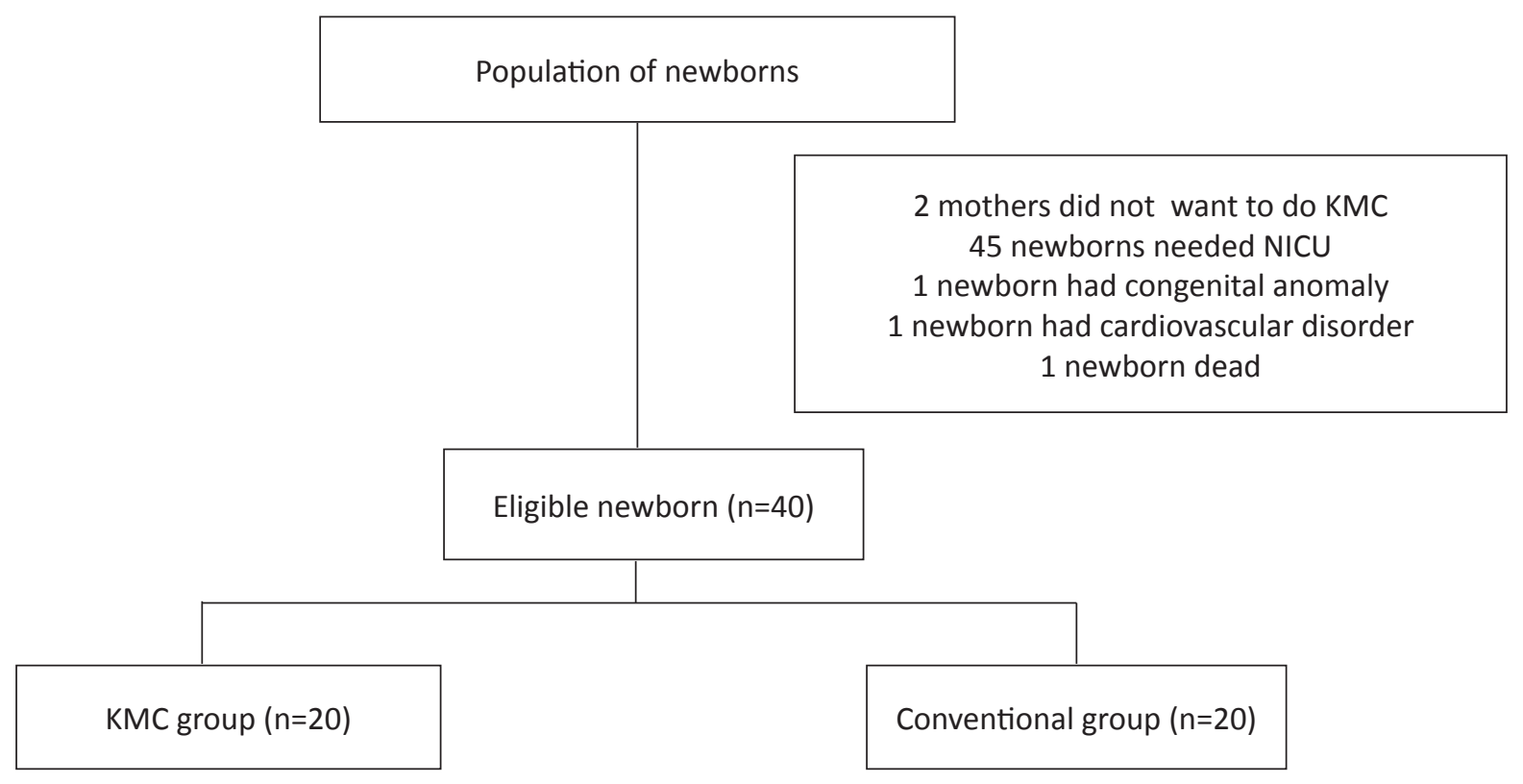

Figure 1: Flow chart of the study

Maternal and fetal demographic characteristics were shown in Table 1. No differences were found between maternal age, parity, gestational age, maternal education, mode of delivery, fetal sex, and the Apgar score between KMC and conventional group. The initially measured weight, length, and head circumferences were similar in both groups ( $p=0.100 ; p=0.353 ; p=0.088$, respectively) making the study results more accurate. The last measured weight was significantly higher in the KMC group than in the conventional group (2187.5 \pm 371.04 vs $1899 \pm$ 242.55; $p=0.015)$. The difference of the mean, the initial and the last weight was also higher in the KMC group than the conventional group $(205.5 \pm 147.451$ vs $96 \pm$ 68.702; $p=0.001$ ). To determine the accuracy of the weight parameter, we calculated the weight gain velocity. The accurate weight gain velocity was $5.098 \pm 2.155 \mathrm{~g} / \mathrm{day}$, and the estimated weight gain velocity was $5.112 \pm 2.168 \mathrm{~g} /$ day. Accurate weight gain velocity, estimated weight gain velocity, and absolute difference percentage were found to be significantly higher in the KMC group than in the conventional group $(p=0.01 ; p=0.009 ; p<0.001)$. But, there were no differences in the last measured weight $Z$ score between both groups $(p=0.364)$.

We also found no differences of the last measured length and head circumference Z-score between both groups (Figure 2). Although the mean difference of initial and last head circumference $(\mathrm{cm})$ showed a significant difference $(p=0.004)$, other parameters regarding head circumferences did not show any difference. Duration of hospital stay was higher in the conventional group than in the KMC group, but this was not significantly different ( $28.4 \pm 5.020$ vs $23.15 \pm 5.184 ; p=0.42$ ). 


\section{D Graph 6}

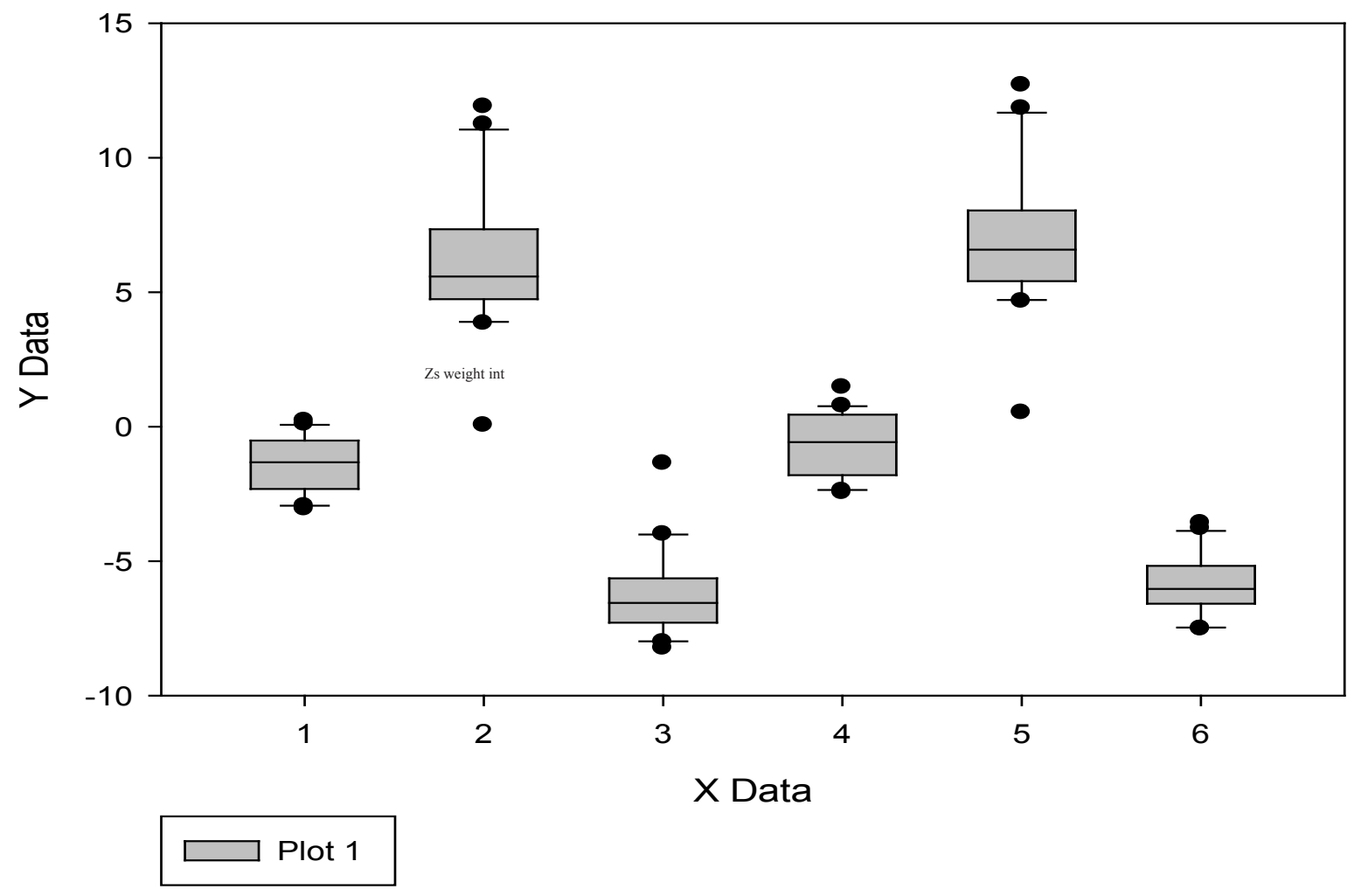

Figure 2: Distribution of Z-score for babies' weight, length, and head circumference (HC) at initial (int) and day (last) 30 measurement

Table 1: Demographic characteristics of maternal, fetal, and measured parameters.

\begin{tabular}{|c|c|c|c|}
\hline Characteristics & KMC group & Conventional group & $\mathbf{p}$ \\
\hline \multicolumn{4}{|l|}{ Maternal characteristics } \\
\hline Maternal age (years old) & $28.6 \pm 2.703$ & $28.7 \pm 4.485$ & 0.263 \\
\hline Parity (n) & $2.25 \pm 0.786$ & $2.1 \pm 0.852$ & 0.765 \\
\hline \multicolumn{4}{|l|}{ Gestational age } \\
\hline$<37$ weeks & 7 (35\%) & $5 \quad(25 \%$ & 0.490 \\
\hline$>37$ weeks & 13 (65\%) & 1575 & \\
\hline \multicolumn{4}{|l|}{ Maternal education } \\
\hline Non-educated & $5 \quad(25 \%)$ & $4 \quad(20 \%)$ & 0.705 \\
\hline Educated & 15 (75\%) & 16 (80\%) & \\
\hline \multicolumn{4}{|l|}{ Maternal morbidity } \\
\hline Preeclampsia & 3 (15\%) & $5 \quad(25 \%)$ & 0.429 \\
\hline Placenta previa & $1(5 \%)$ & $0 \quad(0 \%)$ & \\
\hline Gestational diabetes & $0 \quad(0 \%)$ & $0 \quad(0 \%)$ & \\
\hline Other causes & 16 (80\%) & 15 (75\%) & \\
\hline \multicolumn{4}{|l|}{ Mode of delivery } \\
\hline Spontaneous delivery & 9 (45\%) & $5 \quad(25 \%)$ & 0.185 \\
\hline Vaginal delivery & 11 (55\%) & 15 (75\%) & \\
\hline
\end{tabular}




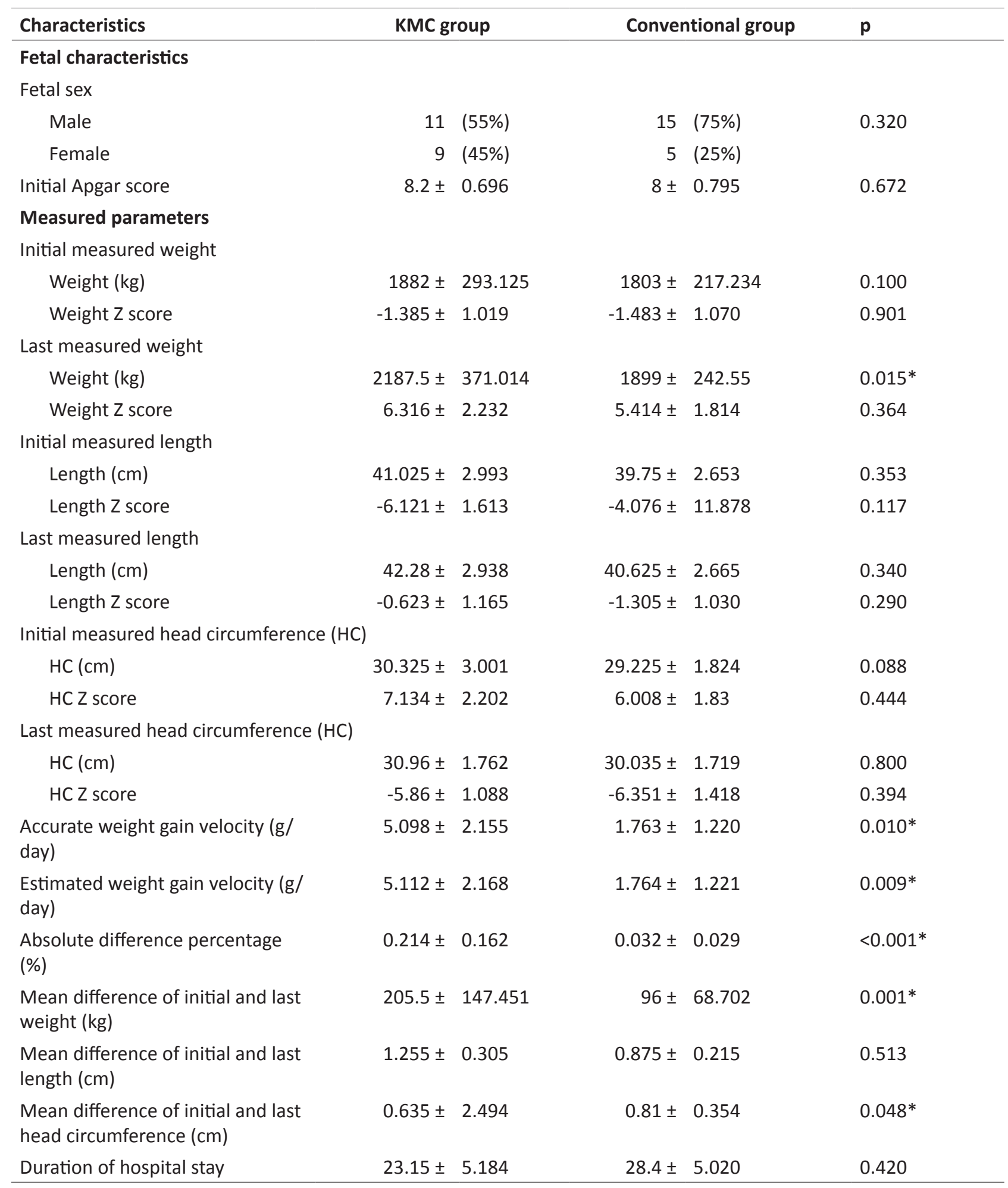

*Significant difference

From the maternal characteristics, only gestational age was found to influence the mean differences of initial and last head circumference $(-0.314 \pm 3.746$ vs $1.415 \pm 1.064$; $p=0.035)$. However, gestational age was not associated with other parameters. There were no differences in maternal age, parity, maternal education, mode of delivery, fetal sex, and initial Apgar score with accurate weight gain velocity, estimated weight gain velocity, absolute difference percentage, and mean differences of initial and last weight, length, and head circumferences (Table 2).

We plotted a mean weight gain graph. Peak weight gains were shown on day 16,18, and 29. Although the graph showed irregular weight gain, on the last day, the increase in weight was greater than the initial weight (Figure 2). 


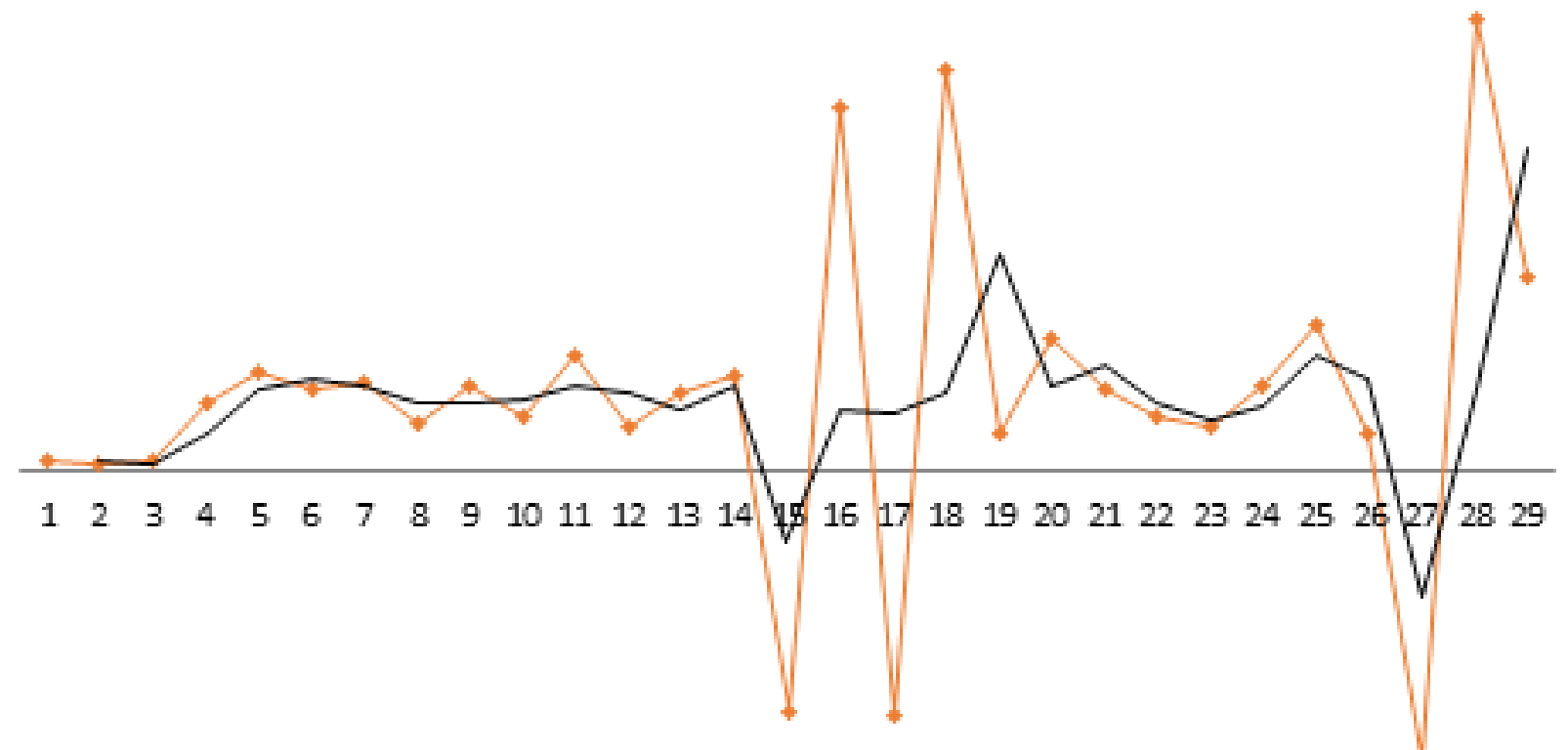

Figure 3: Mean weight gain in KMC group (red line showed the real weight gain and black line showed smoothed out curve)

\section{Discussion}

This is the first study that specifically determined the effect of maternal factors on the successful use the of Kangaroo Mother Care in low birth weight babies compared with conventional therapy. Pratiwi et al. (2015) showed that high levels of maternal education had no effect $(p=0.408)$ (22) and Rodriguez et al. (2007) showed no difference in weight gain with the application of KMC in LBW newborns, whether with spontaneous delivery or cesarean section (23).

The maternal-fetal skin-to- skin contact therapy of KMC is applied as soon as possible after birth (24), helping mothers in their role as providers of biological and psychoemotional needs for their child $(25,26)$. According to WHO guidelines, continuous $\mathrm{KMC}$ is indicated in infants with BBL below $2000 \mathrm{~g}$. Intermittent KMC can also be useful in infants hospitalized in NICU, but this still needs further research (27).

The importance of $\mathrm{KMC}$ is in the enhanced bonding of mother and child. Feldman et al. (2002) successfully demonstrated the positive impact in the relationship between mother and child (28). Athanasopolou et al. (2014) showed that KMC resolved negative moods of maternal anxiety and depression (29). KMC also increased the confidence and competence of the mother to care for her baby and significantly reduced stress. These all contribute to the improvement in the growth and development of the newborns (30).

In this study, an assessment of growth was based on the infant's weight, length, and head circumference, which are the important markers of a child's health $(31,32)$. Bera et al. (2014) showed that in infants receiving KMC, the growth parameters and mental development were better than in infants who were treated conventionally (33).
However Ali et al. (2009) showed that infants treated in an incubator had a higher weight gain per day (19.3 vs.10.4 g, $p<0.001)$, shorter duration of stay $(6.9 \%$ vs. $23.2 \% p=$ $0.014)$, lower infection rate (6.9\% vs. $23.2 \% p=0.014)$, than babies who received KMC (19). Palencia et al. (2009) in 115 LBW infants who were followed up until 13 months, showed that the growth in height for age was higher within the percentiles of weight for age $(p=0.0001)$. Male gender had a higher weight than females $(p=0.031)$ (34). This study was similar to the study in Indonesia reported previously. In that study, we showed that only the last measured weight, the difference in the initial and the last weight, and the weight gain velocity were higher in the KMC group than the conventional group. No differences were found in the length and head circumferences. Haksari (2004) and Rahmayanti (21) in Indonesia also found that there was no difference between weight/age, length/age, head circumference/age in LBW infant treated with KMC and conventional therapy. On the other hand, Rao et al. (20) showed that infants treated in incubators had a higher weight gain, head circumference ( $0: 49$ vs $0.75 \mathrm{~cm}, p=0: 02)$, and body length ( 0.99 vs. $0.7 \mathrm{~cm}, \mathrm{p}=0.008$ ) compared with infants who received KMC.

It had been observed that weight gain should approximate an intrauterine growth rate of 16.8 to $30.7 \mathrm{~g} /$ day (32). In this study, the accurate weight gain velocity was 5.098 $\pm 2.155 \mathrm{~g} /$ day and the estimated weight gain velocity was $5.112 \pm 2.168 \mathrm{~g} /$ day. These were still far below the recommended growth rate. The Z-score of weight was still below the expected average. Newborns were still categorized as small for gestational age at the end of the study.

The parameters of weight and length were not affected by maternal age, parity, maternal education, mode of delivery, fetal sex, and the initial Apgar score. Newborns 


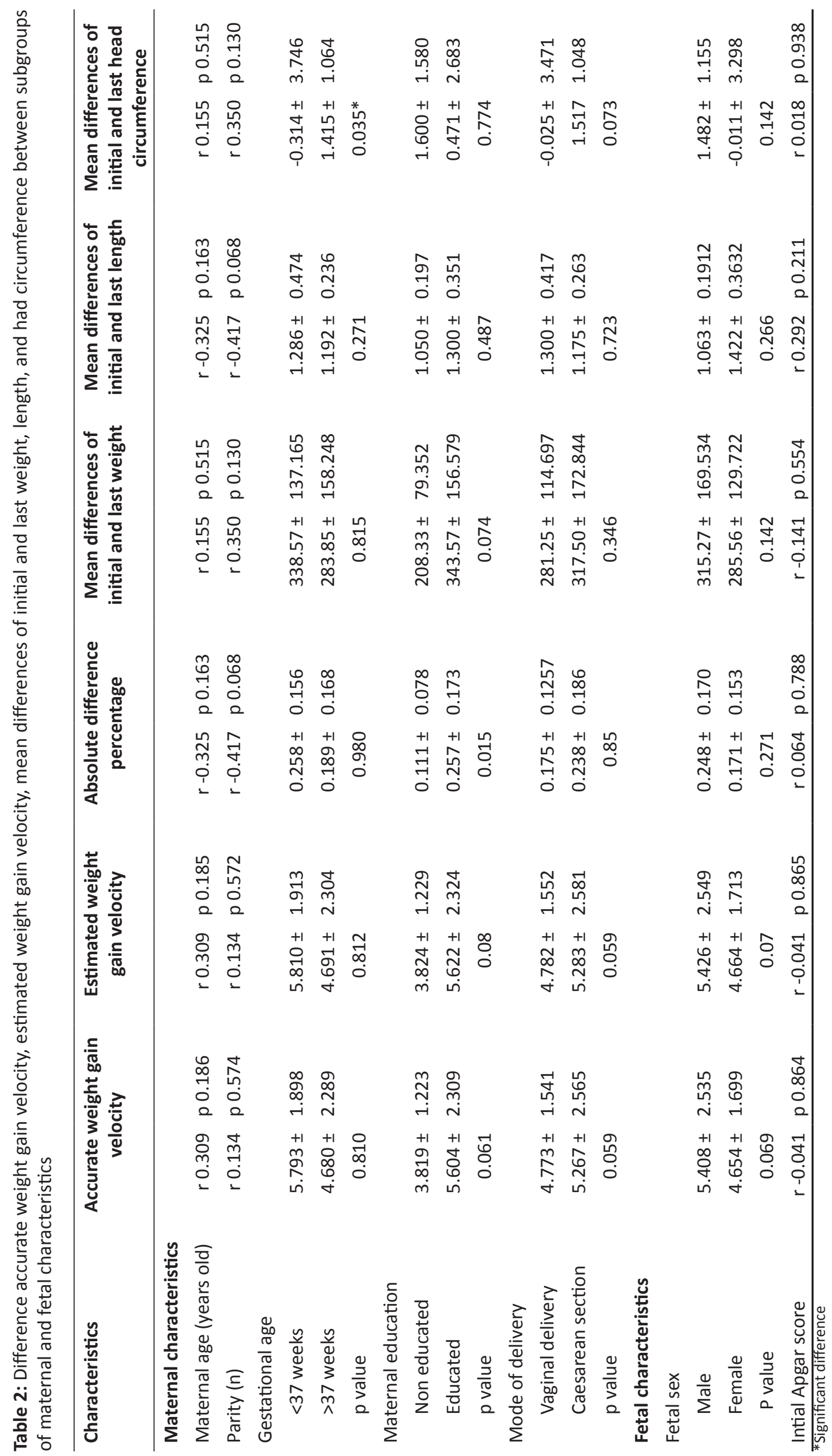


at birth who were $<37$ weeks of gestational age, had delayed the growth of head circumference. KMC had been proved to increase cerebral blood flow, acting as nutritional support, mainly to the brain. Korraa et al. (2014) found that newborns who received KMC had a lower cerebral blood flow resistive index $(p<0.05)$, indicating an improved cerebral blood flow (35). The influence of immature organ in preterm babies need to be considered, and this would require further research (36).

The strength of this study is the detailed follow-up parameters which are better than those reported in other studies. The better follow-up rate in the KMC group could be due to the active involvement of the mother in the care of her LBW baby. The limitations of this study are the small sample size conducted in two institutions only. Further research is needed to confirm these findings.

\section{Conclusion}

There were no maternal and fetal differences in the growth parameters of the two groups of the study, except for the delayed growth of head circumferences in preterm infants.

\section{Acknowledgements}

I am greatly appreciate to all observers and my students who had directly or indirectly involved in supporting me to finish this study.

\section{Conflict of interest}

The author declared no conflict of interest in this study.

\section{References}

1. Beddek F, Demmouche A, Mai AH, Ghani A \& Benali A. Low birth weight in Sidi Bel Abbes region (west of Algeria): retrospective study of 10008 deliveries. J Blood Disorders Transf 2014; 5: 1-4.

2. United Nations Children's Fund and World Health Organization, Low Birthweight: Country, regional and global estimates. UNICEF, New York, 2004. p.1

3. Kumari R, Guduri GB, Venkateswarulu. A study on maternal factors affecting low birth weight in institutional deliveries. IOSR J Dental Med Sci. 2015; 14(1): 45-48.

4. Nagargoje M, Chaudhary S, Deshmukh J, Gupta S, Misra S. A Case Control Study Of Risk Factors For Low Birth Weight in Nagpur City Of Maharashtra. Indian J Community Health. 2011; 22(2, 1): 4-7.

5. Azimul S, Matin A, Shabnam J, Shamianaz S, Bannerje M. Maternal factors affecting low birth weight in urban area of Bangladesh. Journal of Dhaka Medical College. 2009; 18(1): 64-9.

6. Jackson HD, Wei Y, Chen F. Quantitative data analysis of multiple factors associated with low birth weight in Bibb country, Georgia. Journal of the Georgia public health association. 2008; 1(1): 24-32.
7. Agarwal K, Agarwal A, Agrawal V, Agrawal P, Chaudhary V. Prevalence and determinants of "Low Birth Weight" among institutional deliveries. Annals of Nigerian Medicine. 2011; 5(2): 48.

8. Mumbare SS, Maindarkar G, Darade R, Yenge S, Tolani MK, Patole K. Maternal risk factors associated with term low birth weight neonates: a matched-pair case control study. Indian Pediatr. 2012; 49(1): 25-8.

9. Taha E, Mohamed Ahmed A, Gadir KN, Mukhtar Y, Yousif E, El-Sadig E. Investigation of the Risk Factors of Low Birth Weight (LBW) in the National Ribat University Hospital, Khartoum. Sudanese Journal of Public Health. 2012; 7(4).

10. Negi KS, Kandpal SD \& Kukreti M. Epidemiological factors affecting low birth weight. JK Science 2006; 8(1): 31-134.

11. Trihardiani I. Faktor risiko kejadian BBLR di wilayah kerja puskesmas singkawang timur dan utara kota singkawang. Available at: https://core.ac.uk/ download/files/379/11731574.pdf

12. Atikah Proverawati dan Siti Misaroh. Nutrisi Janin dan Ibu Hamil. Yogyakarta: Nuha Medika: 2010. Hal 88-148

13. Erica RE, Armstrong S. Pencegahan Kematian Ibu Hamil. Jakarta: Binarupa Aksara; 1994. Hal 40-41

14. WHO: Kangaroo mother care: a practical guide. World Health Organization: Geneva, Switzerland 2003.

15. Alisjahbana A, Usman A, Irawati S, Triyati A. Prevention of hypothermia of low birth infants using kangaroo method. Pediatr Indones 1998; 38:205-14

16. Whitfield MF, Grunau RE. Behavior, pain perception, and the extremely low-birth weight survivor. Clin Perinatol. 2000; 27(2):363-79.

17. Martínez H, Rey E, Navarrete L, Marquette C. Programa Madre Canguro en el Instituto Materno Infantil de Bogotá. Primer Encuentro Internacional del Programa Madre Canguro Bogotá: UNICEF. 1990:21-43.

18. Whitelaw A, Sleath K. Myth of the marsupial mother: home care of very low birth weight babies in Bogota, Colombia. The Lancet. 1985; 325(8439):1206-8.

19. Ali SM, Sharma J, Sharma R, Alam S. Kangaroo mother care as compared to conventional care for low birth weight babies. Dicle Medical Journal/Dicle Tip Dergisi. 2009; 36(3):155-160.

20. Suman Rao P, Udani R, Nanavati R. Kangaroo mother care for low birth weight infants: a randomized controlled trial. Indian pediatr. 2008; 45(1):17.

21. Rahmayanti SD. Influence of treatment methods kangaroo on the growth of the baby, knowledge, and attitude of a mother in caring for LBW in hospitals Cibabat Cimahi. FIK UI 2010.

22. Pratiwi R, Kosim MS, Wijayahadi N. Prognostic factors for success in the Kangaroo Mother Care method for low birth weight babies. Paediatr Indones. 2015; 55(3):143.

23. Rodriguez A, Nel M, Dippenaar H, Prinsloo E. Good short-term outcomes of kangaroo mother care in 
infants with a low birth weight in a rural South African hospital. S Afr Fam Pract. 2007; 49(5):15-c.

24. Charpak N, Ruiz-Peláez JG, Charpak Y. A randomized, controlled trial of kangaroo mother care: results of follow-up at 1 year of corrected age. Pediatrics. 2001; 108(5):1072-9.

25. Kadam S, Binoy S, Kanbur W, et al. Feasibility of kangaroo mother care in Mumbai. Indian J Pediatr. 2005; 72(1):35-38.

26. Ludington-Hoe SM, Morgan K, Abouelfettoh A. A clinical guideline for implementation of kangaroo care with premature infants of 30 or more weeks' postmenstrual age. Adv Neonatal Care. 2008; 8(3):S3-S23.

27. WHO: Kangaroo mother care: a practical guide. World Health Organization: Geneva, Switzerland 2003.

28. Feldman R, Eidelman Al, Sirota L, Weller A. Comparison of skin-to-skin (kangaroo) and traditional care: parenting outcomes and preterm infant development. Pediatrics. 2002; 110(1):16-26.

29. Athanasopolou E \& Fox JR. Effects of kangaroo mother care on maternal mood and interaction patterns between parents and their preterm, low birth weight infants: a systematic review. Infant Mental Health J. 2014; 35(3):245-262.

30. Tessier R, Cristo M, Velez S, Girón M, de Calume ZF, Ruiz-Paláez JG, et al. Kangaroo mother care and the bonding hypothesis. Pediatrics. 1998; 102(2):e17-e.
31. Kaushal SK, Misra SK, Gupta SC \& Singh R. A study of maternal factors and birth weight in a border district of uttar pradesh: a hospital based study. Indian J Community Health. 2012; 24(2):86-90.

32. Weller A, Rozin A, Goldstein A, et al. Longitudinal assessment of pituitary thyroid axis and and renal function in preterm infants raised by "kangaroo mother care". Horm Res 2002; 57(1-2):22-6.

33. Bera A, Ghosh J, Singh AK, Hazra A, Mukherjee S \& Mukherjee R. Effect of kangaroo mother care on growth and development of low birthweight babies up to 12 months of age: a controlled clinical trial. Acta Paediatr 2014; 103(6): 643-650.

34. Palencia D, Mendoza CJ, Torres J \& Echandia CA. Kangaroo mother program: physical growth and morbidity in a cohort of children, followed from 40 weeks of postconceptional age until first year. Colombia Medica. 2009; 40(3):292-9.

35. Korraa AA, El Nagger AA, Mohamed RAE-S, Helmy NM. Impact of kangaroo mother care on cerebral blood flow of preterm infants. Ital J Pediatr. 2014; 40(1):1.

36. Goldstein RF. Developmental care for premature infants: a state of mind. Pediatrics. 2012; 129(5):e1322-e3. 